\title{
CORRECTION
}

\section{Correction to: Thrust measurements and evaluation of asymmetric infrared laser resonators for space propulsion}

\author{
O. Neunzig ${ }^{1} \cdot$ M. Weikert ${ }^{1} \cdot$ M. Tajmar $^{1}$
}

Published online: 27 May 2021

(c) The Author(s) 2021

\section{Correction to: CEAS Space Journal https://doi.org/10.1007/s12567-021-00366-4}

Original version contains incorrect Table 3 values. Correct Table 3 values are provided through this correction.

Table 3 LED cavity (BART drive) measurements

\begin{tabular}{llll}
\hline Setup & Measurement influence & Power (P) & Measured thrust \\
\hline BART drive & None (Differential) & $770 \mathrm{~mW}$ & $(0.13 \pm 1.35) \mathrm{nN}$ \\
& & $1540 \mathrm{~mW}$ & $(0.22 \pm 4.13) \mathrm{nN}$ \\
\hline
\end{tabular}

Publisher's Note Springer Nature remains neutral with regard to jurisdictional claims in published maps and institutional affiliations.

The original article can be found online at https://doi.org/10.1007/ s12567-021-00366-4.

M. Tajmar martin.tajmar@tu-dresden.de

O. Neunzig

oliver.neunzig@tu-dresden.de

M. Weikert

marcel.weikert@tu-dresden.de

1 Institute of Aerospace Engineering, Technische Universität Dresden, Marschnerstrasse 32, 01307 Dresden, Germany 